\title{
DNA-Like Duplex Structures Derived from Chemistry Based on 2'-Deoxy-Cytidine: A New Model for Base-Specific Inhibition of $G$ and $C$ on DNA and RNA Level
}

\author{
Henk M. Buck \\ Kasteel Twikkelerf 94, Tilburg, The Netherlands \\ Email: h.m.buck@ziggo.nl
}

How to cite this paper: Buck, H.M. (2018) DNA-Like Duplex Structures Derived from Chemistry Based on 2'-Deoxy-Cytidine: A New Model for Base-Specific Inhibition of $\mathrm{G}$ and $\mathrm{C}$ on DNA and RNA Level. Journal of Biophysical Chemistry, 9, 23-40. https://doi.org/10.4236/jbpc.2018.93003

Received: December 3, 2018

Accepted: December 26, 2018

Published: December 29, 2018

Copyright $\odot 2018$ by author(s) and Scientific Research Publishing Inc. This work is licensed under the Creative Commons Attribution International License (CC BY 4.0).

http://creativecommons.org/licenses/by/4.0/

(c) (i) Open Access

\begin{abstract}
The new epigenetic elements 5-hydroxymethyl-dC, 5-formyl-dC, and 5-carboxy- $\mathrm{dC}$ may be considered as intermediates of an active demethylation process. A comprehensive mechanistic model is given for the $\mathrm{C}-\mathrm{C}$ bond cleavage focused on the chemistry within the DNA duplex structure. In addition we register spin-off chemistry of this process in evaluating new duplex systems closely related to natural DNA and RNA concerning their hydrogen-bond symmetrization. A model is composed for a base-specific inhibition of $\mathrm{G}$ and $\mathrm{C}$ on the DNA and RNA level. C-G combinations are of general importance in controlling the dynamics of gene expression. In some way the suggested model systems are related to antisense oligonucleotides (ASOs).
\end{abstract}

\section{Keywords}

Active Demethylation, DNA Catalysis, Symmetry Elements,

(Anti)Parallel Modified DNA and RNA, Inhibition of Genetic Information

\section{Introduction}

It may be of interest to focus our attention specifically on the base manipulation as shown in recent studies related to the DNA conformation, especially at DNA systems for B-Z transition and their significance as epigenetic model [1]. Possibly, there is a consistency with a study with regard to the effect on the B-Z transition of the three different cytosine modifications i.e. 5-hydroxymethyl, 5-formyl, and 5-carboxyl as derived from 5-methyl cytosine, mediated by the TET (ten eleven translocation) family of enzymes, and known as new DNA bases 
on $\mathrm{C}_{\mathrm{P}} \mathrm{G}$ dinucleotide sequences [2] [3] [4]. It was demonstrated that 5-hydroxymethylcytosine inhibits the $\mathrm{B}-\mathrm{Z}$ transition, whereas the other bases facilitate this conversion [5]. At the same time a study showed the significance of 5-formylcytosine in altering the structure of a DNA double helix [6]. With X-ray the crystal structure of a DNA dodecamer with three 5-formyl $C_{P} G$ units shows a clear change in the conformation of the grooves resulting in helical underwinding, indicated as F-DNA. Hydrogen reduction results in 5-hydroxymethylcytosine under formation of a B-DNA structure. An interesting aspect is that the increase of concentrations of spermine results in conversion of the 5-formylcytosine containing oligomers into B-DNA. Apparently shielding of regiospecific phosphate anions plays an important role in this conformational change. It may be suggested to introduce 5-substituted cytosines with phosphate-methylated backbones $\left(\mathrm{POCH}_{3}\right)$ in these studies, as was done with the Z-conformation of the phosphate-methylated $d\left(C_{p} G\right)$. Even on the dimer level information will become available to predict the conformational behavior of corresponding long oligomers [7]. It may be helpful to investigate the parameters which might be responsible for the different conformations of DNA and RNA with respect to backbone- and base-manipulation. Methylation of the phosphate backbone inhibits the coordination via hydrogen networks with the phosphate backbone as present under natural conditions. From the output of the above-mentioned results it is evident that DNA may be a fascinating template for interaction with a diversity of molecular systems, vide infra. Generally, the focus on the new epigenetic compounds is based on the oxidation via TETenzymes of 5-methyl-deoxycytidine (mdC) to 5-hydroxymethyl-dC(hmdC), 5-formyl-dC(fdC), and 5-carbo$\mathrm{xy}-\mathrm{dC}(\mathrm{cadC})$ as shown in Figure 1.

From the new cytosine compounds which were found in stem cell DNA, it was assumed that these 5-substututed ones may be considered as intermediates of an active demethylation process [8]. This process may include excision repair, $\mathrm{C}-\mathrm{C}$ bond cleavage or deamination of hmdC into hmdU. Recent experiments of Iwan et al. clearly demonstrated that with the incorporation of isotope labeled $\mathrm{fdC}$ into the genome of cultured mammalian cells, the formation of the unmodified $\mathrm{dC}$ can be explained by C-C bond cleavage [9].

At the same time we published an explanation for the exchange of information between these epigenetic bases and the DNA backbone via intramolecular phosphorylation in the case of hmdC under formation of $\mathrm{dC}$ and formaldehyde. The role of phosphorus in an activated state as trigonal bipyramidal intermediate shows, as demonstrated by model systems, its catalyzing function in the dehydroxymethylation, deformylation, and decarboxylation [10].

The present paper is focused on the dynamics and molecular mechanism of the $\mathrm{C}-\mathrm{C}$ bond cleavage as vital part of the suggested in vivo mechanism. Moreover, we will give attention to spin-off chemistry of the $\mathrm{C}-\mathrm{C}$ bond cleavage with an introduction of hydrogen-bond bridging for the synthesis of new duplex systems and a model consideration of base-specific inhibition for guanine and cytosine on the DNA and RNA level. 

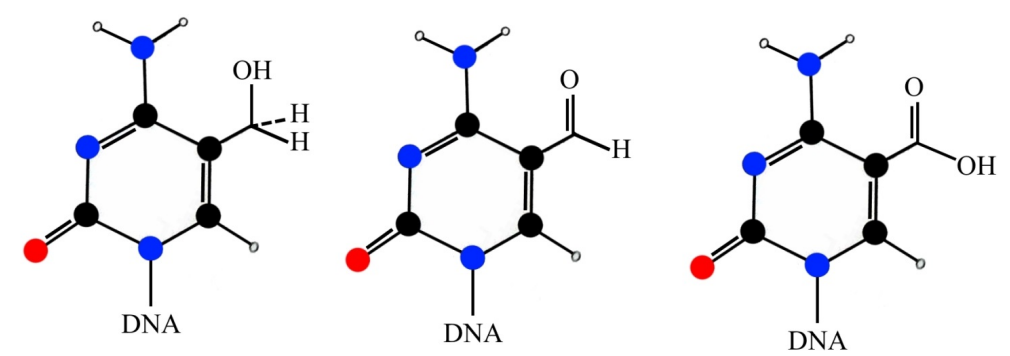

Figure 1. Structures of 5-hydroxymethyl-dC(hmdC), 5-formyl-dC(fdC), and 5-carboxy-dC(cadC).

\section{Results and Discussion}

\subsection{Theoretical Approach}

A model study showed that the C-G pairing in the DNA duplex has an inversion center concerning the hydrogen-bridging part of the coupled bases as compared with the other combination T-A, see Figure 2 and Figure 3, respectively. We suggest that this particular mode of symmetry-related interaction may lead to the synthesis of duplexes with installed physical-chemical and biochemical properties. For the T-A pair we recognize rotamers around the N3-C6 link (T) and the N1-C4 link (A) separately. The absence of these rotamers in the combination $\mathrm{C}-\mathrm{G}$ implies a specific role for the presence of an extra carbonyl group (T)in the absence of an amino group at C2 (A) necessary for the formation of a third hydrogen bridge as would be the case for 2,6-diaminopurine as substitute for $A$. This general view introduces a certain degree of genetic imperfection in DNA. In the case of T-A we are dealing with the lack of a hydrogen bridge, compared to $\mathrm{C}-\mathrm{G}$, in combination with the introduction of a conformational instability caused by the extra carbonyl group. In our opinion these considerations are also applicable on the RNA level of hybridization. In that case $\mathrm{T}$ is replaced by $\mathrm{U}$. The missing of the $5-\mathrm{CH}_{3}$ group in $\mathrm{U}$ will have a beneficial effect on the spatial interaction in the case of a rotameric conformational change. It must be emphasized that this approach is based on certain symmetry elements present in these genetic systems. In order to promote or suppress these particularities in the duplex requires control of specific interactions with well-organized systems as complex networks of interacting proteins and enzymes.

With the model-based approach we could introduce a specific inhibition of $\mathrm{G}$ and $\mathrm{C}$ on a level different from the usual modified site specific DNA and RNA model compounds. This aspect will be further described under the latter two sections of Results and Discussion.

Based on recent work of Schiesser et al. with respect to the $\mathrm{C} 5=\mathrm{C} 6$ bonds of $\mathrm{hmdC}, \mathrm{fdC}$, and cadC (see Figure 1) in the presence of thiols as biologically relevant (organo) catalyst [12], we recognized the impact of the Michael-addition reaction triggering the reconversion of $\mathrm{dC}$.

For a molecular differentiation between duplex and individual bases we selected the sophisticated geometric $a b$ initio calculations of the corresponding 

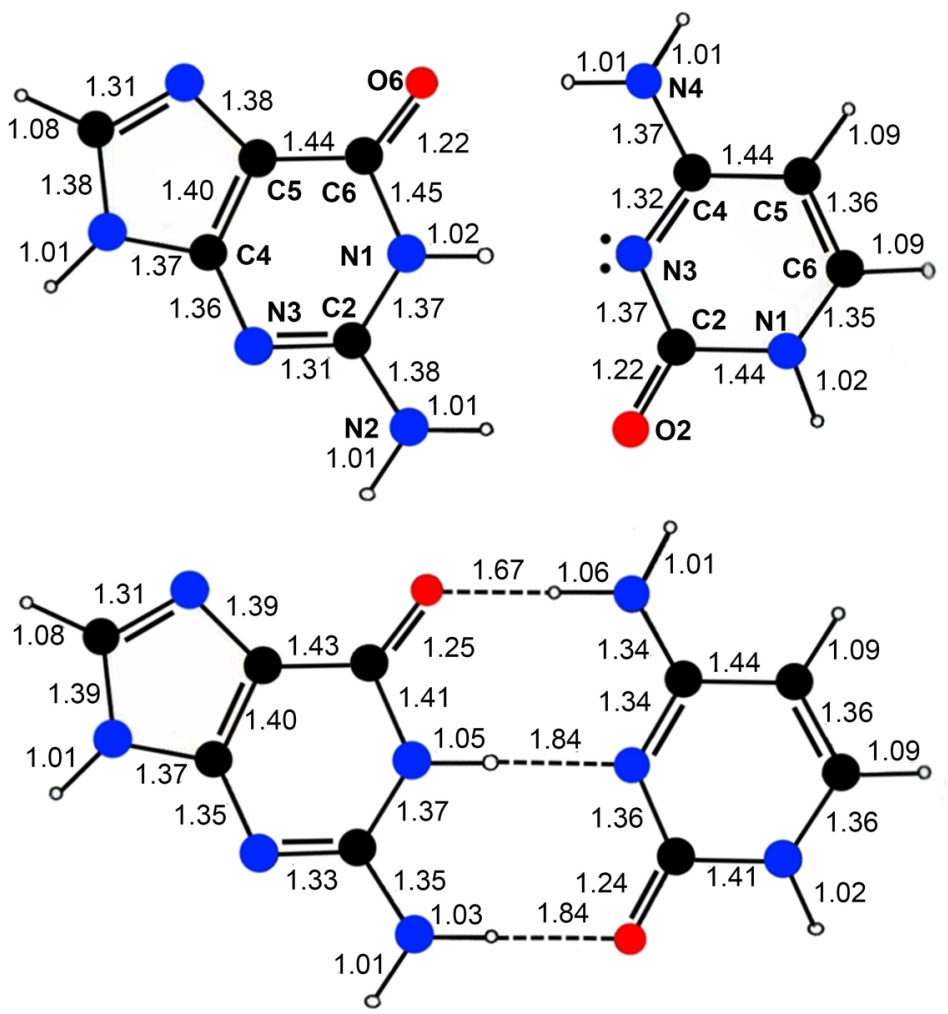

Figure 2. Structure of the Watson-Crick pair C-G with bond distances ( $)$. The geometric parameters and visualization were obtained from the thesis of Fonseca Guerra [11].
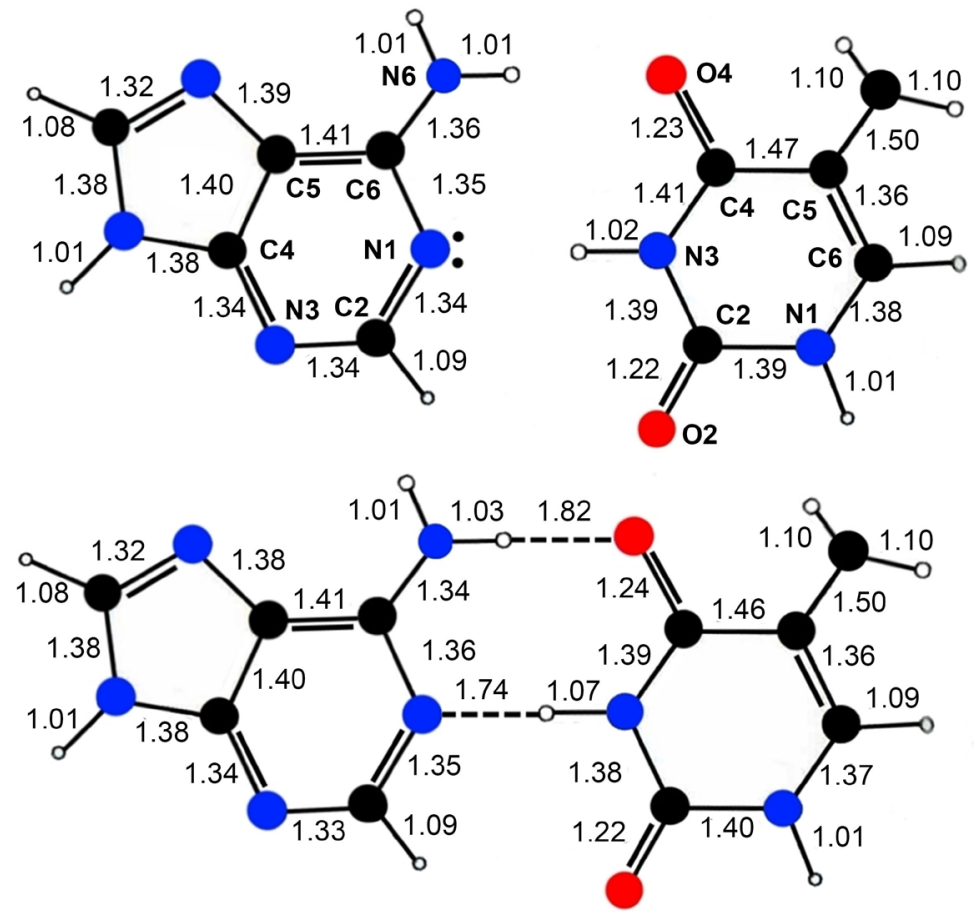

Figure 3. Structure of the Watson-Crick pair A-T with bond distances ( $)$. The geometric parameters and visualization were obtained from the thesis of Fonseca Guerra [11]. 
duplex DNA and the separate structures of Fonseca Guerra et al. for a realistic comparison [13]. With this presentation the different local symmetry elements are more clear and accompanied with realistic modes for intramolecular dynamics. This approach opens up new routes for making use of DNA not only as a unique model for storage and transferring of genetic information but also as a template for chemistry and catalysis thereby making use of the various sites and groups with different reactivity and the specific hydrogen bridging in the CG duplex. In fact the same mechanisms may play their role in DNA-RNA and RNA-RNA (modified) structures. With regard to the influence of the various backbone manipulations on the (de)stability of DNA-DNA, RNA-RNA, and DNA-RNA duplexes, we took profit of the phosphorus chemistry in combination with its varying coordination in preparing modified anti-sense and sense model systems (generally indicated as ASOs) by methylation of the phosphate groups thereby introducing chirality in the backbone that could result in (un)favorable conformations for duplex formation. Generally, phosphate-methylation influences the conformation of the sugar ring. Under this condition phosphate-methylated DNA (fixed B-conformation) hybridizes with its natural complementary DNA (A-and B-conformation) strand and only for a small sequence range in hybridization with the corresponding natural RNA strand (fixed A-conformation) [14]. Phosphate-methylated RNA's (A-conformation) were prepared in a similar way as the phosphate-methylated DNA's suggesting a related hybridization with natural DNA and RNA complements. In the case of pyrimidine bases (anti)parallel DNA and RNA duplexes were formed in which the phosphorus chirality is very dominant. Even under natural conditions (peptide induced) similar observations were made [15]. In these systems the role of the protein has a stereoselective character that reflects in a certain way the combination of the phosphate shielding with a stereochemical fit with the duplex. This process of bioconjugation may be considered as an accommodation of autonomic properties resulting in an unexpected duplex form. This summary corresponds perfectly with the todays requirements for ASOs as has been recently explained particularly for the treatment of spinal muscular altrophy [16].

The latter successful therapy is based on 18 nucleosides bound by phosphorothioate (PS) linkages in which one of the non-bonding oxygen atoms is replaced by sulfur. In contrast with the natural phosphodiester linkages the phosphorothioate modification demonstrates a high degree of metabolic stability and an improved cellular uptake [17]. Similar observations have been mentioned by us for phosphate-methylated DNA and RNA. These systems show an additional property based on the absence of phosphate-phosphate repulsion of the two strands between the modified DNA and RNA and their complementary strand i.e. increase duplex stability. The impact of the phosphorus chirality on the conformational behavior and corresponding duplex stability has been described, vide supra.

From recent literature it can be concluded that DNA has become a tool for the 
preparation and construction of highly ordered materials making use of the well-known locked nucleic acids based on their rigidity and their increased ability for duplex formation [18]. A combination of properties is in excellent agreement with the biophysical properties of phosphate-methylated DNA and RNA.

Using the Watson-Crick duplex structure, we assume that our model delivers a contribution to the molecular mechanism of the $\mathrm{C}-\mathrm{C}$ bond cleavage of $\mathrm{C} 5-\mathrm{CH}_{2} \mathrm{OH}, \mathrm{C} 5-\mathrm{C}(\mathrm{O}) \mathrm{H}$, and $\mathrm{C} 5-\mathrm{C}(\mathrm{O}) \mathrm{OH}$ within the duplex structure for the demethylation process, vide infra. It should be mentioned that in the representations as shown in Figure 2 and Figure 3 there was chosen for shielding of the phosphate anions by protonation [13]. In an elaborate study of the (bio)chemistry of phosphate-methylated DNA the significance of complete phosphate shielding was experimentally and theoretically described in a number of papers, demonstrating the unique (bio)chemical differences between natural and phosphate-methylated DNA, vide supra. The absence of the correct environment of water and counter ions as $\mathrm{Na}^{+}$etc. finds more or less a certain correction in proton shielding though with the negative aspect of the introduced complete proton shielding of the phosphate anions.

Generally, our model description clearly shows the role of hydrogen bridging as is demonstrated by the phosphate shielding of the 5-formyl-deoxycytidines in the duplex of a DNA dodecamer comprising three $5 \mathrm{fCpG}$ sites, resulting in helical under winding [6].

\subsection{C-C Bond Cleaving Reactions via a Michael Addition}

The $\mathrm{hmdC}, \mathrm{fdC}$, and cadC nucleosides as shown in Figure 1 were incorporated in oligodeoxynucleotides using the solid phase phosphoramidite chemistry or the PCR procedure [19] [20] [21] [22]. The 20-mers were constructed as hairpin loop structures containing four T's whereas the remaining part forms the double-stranded deoxynucleotides containing either hmdC, fdC or cadC. The $\mathrm{C}-\mathrm{C}$ bond cleavage was obtained in a high concentration of thiols and under specific $\mathrm{pH}$ conditions. This concept was based on the experimental data that thiols are active side residues in DNA modifying enzymes [23].

With these results in mind we focus on the fragments C6-C5-C4-N3 of the pyrimidine bases as shown in Figure 2 and Figure 3 for the single and dimer structures because the suggestion was put forward that a helper nucleophile (thiol) initiates at $\mathrm{C} 6$ a Michael-addition reaction resulting in dehydroxymethylation, deformylation, and decarboxylation with yields of $0.5 \%, 2.5 \%$, and $28 \%$, respectively [12]. It was established that the rate of conversion of hmdC in contrast to $\mathrm{fdC}$ and cadC is a slow process. Apparently the leaving capacity of the introduced substituents as formaldehyde, formic acid, and carbon dioxide plays a role in the overall conversion.

For that process the C6-C5-C4-N3 fragment of cytosine is the favored route. Accepting this model, the significance of the duplex structure is promoted by its ability of hydrogen bridging between N1-H1---N3 of guanine and cytosine respectively, thereby stabilizing the electron displacement by (partial) lone-pair 
fixation on N3. The displacement of bonds is shown in Figure 4.

After activation of the $\mathrm{C}-\mathrm{G}$ plateau by the nucleophilic addition of a thiol as given in Figure 4, the cytosine fragment N3-C4-C5 is negatively charged resulting in $\mathrm{C} 5$ protonation. Summarizing, we introduce a modified model for the activation of the system for the $\mathrm{C}-\mathrm{C}$ bond cleaving in which the DNA duplex structure is incorporated controlling with hydrogen bridging the displacement of the negative charge. This specific chemistry within the double-stranded DNA may contribute to the knowledge on the dynamics of proton transfer between the bases cytosine and guanine involved.

Such dynamics was recently observed for quantum hydrogen bond symmetrization under high pressure. Under these conditions the symmetrization of the hydrogen bond in ice is realized indicating that the hydrogen-bonded protons initially located at asymmetric positions of the $\mathrm{O}-\mathrm{O}$ separation will relocate to the symmetric midpoint [24]. A similar observation has been recently obtained for hydrogen sulfide under high pressure with $\mathrm{H}_{3} \mathrm{~S}$ stoichiometry creating an impressive network of hydrogens. As in the former case the asymmetric location of the hydrogens with respect to sulfur changes in a symmetric location under high-pressure conditions. The latter symmetry change in $\mathrm{H}_{3} \mathrm{~S}$ is accompanied with superconductivity [24] [25].

It seems very unlikely that corresponding symmetry hydrogen bonding occurs in natural systems as DNA. Theoretical and experimental studies on structural changes in DNA under relatively high external pressure resulted in (very) small distortions focused on the hydrogen bond lengths [24]. Errea et al. demonstrated clearly that under high-pressure conditions the possibility for proton displacement is opened under reduction of the transition between asymmetric and symmetric structures. In order to investigate these various aspects for more sophisticated biological systems, we are limited in our choice concerning the intrinsic symmetry elements, vide infra.

Concerning the T-A combination, the $\mathrm{C} 6-\mathrm{C} 5$ has double bond character necessary for initiating the Michael-addition reaction. However, the electron-accepting site is the $\mathrm{C} 4=\mathrm{O}$ group (see Figure 3 ) which can be considered as a dead end, reinforced by the absence of double bond character of C4-N3.

The mechanism for the $\mathrm{C}-\mathrm{C}$ bond cleavage as proposed by Schiesser et al. follows a dynamic procedure [12]. However, it lacks structural necessities corresponding with the well-known elimination E1 and E2 profiles for such conversions. Starting with $\mathrm{hmdC}$, the $\mathrm{C} 5-\mathrm{CH} 2 \mathrm{OH}$ bond is poorly activated for the initiation of the $\mathrm{C} 5=\mathrm{C} 6$ bond in cytosine. For the overall process it means elimination of the $\mathrm{CH}_{2} \mathrm{OH}^{+}$cation under formation of formaldehyde. For the other compounds, the reactivity proceeding in the suggested direction will be strongly stimulated by carbonyl hydration indicated by change in the initial substitution for $\mathrm{X}$. With the intermediate presentation in Figure 5, as follows after protonation and shown in Figure 4, the restoring of the $\mathrm{C} 5=\mathrm{C} 6$ bond can be demonstrated by the electron displacement under formation of $\mathrm{X}^{+}$, the short-living $\mathrm{CH}(\mathrm{OH})_{2}$ and $\mathrm{C}(\mathrm{OH})_{3}$ carbocations, and the thiolate anion $\mathrm{RS}^{-}$. In fact this is a 

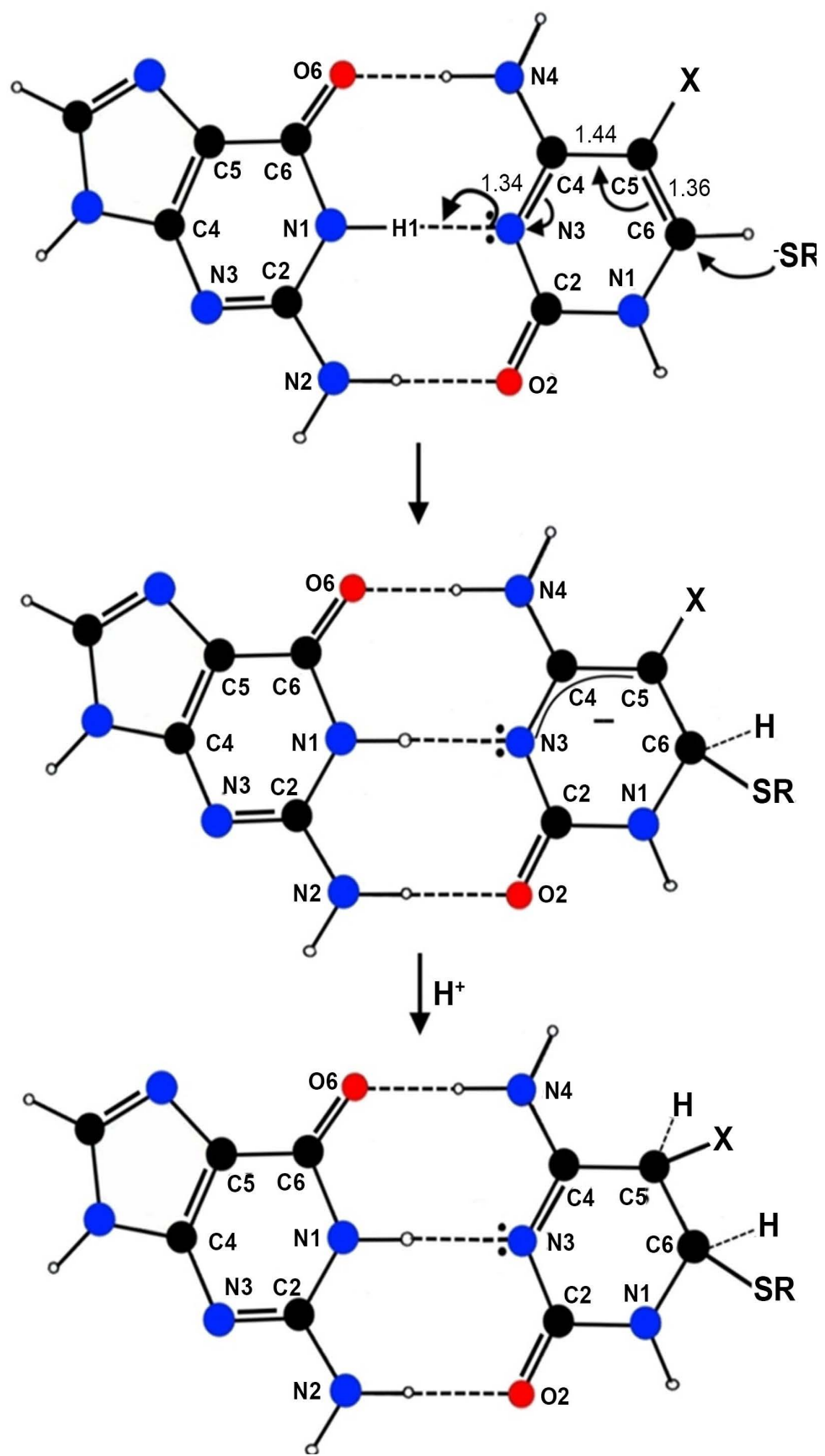

Figure 4. Dynamic and mechanistic picture of a nucleophilic addition of a thiol (RSH) on the $\mathrm{C} 6=\mathrm{C} 5$ bond of 5 -substituted (X) cytosine with $\mathrm{X}=-\mathrm{CH}_{2} \mathrm{OH}$, $-\mathrm{C}(\mathrm{O}) \mathrm{H}$, and $-\mathrm{C}(\mathrm{O}) \mathrm{OH}$, catalyzed by providing partial lone-pair shielding of $\mathrm{N} 3$ with the N1-H1 bond of guanine. The overall description results in the crucial intermediate phase in which C5 is protonated.

nice demonstration for a simple conversion of an electron accepting group into an electron donating group illustrating the importance for biomolecular concepts in enzymatic processes.

As mentioned for $\mathrm{fdC}$ and cadC, the reactivity proceeding in the suggested direction will be strongly stimulated by carbonyl hydration as indicated by 
change in the initial substitution for $\mathrm{X}$ in Figure 5. Therefore in these cases we suggest $\mathrm{S}_{\mathrm{N}} 1$-like conversions, under the formation of formic acid and carbon dioxide, via the short-living $\mathrm{HC}(\mathrm{OH})_{2}^{+}$and $\mathrm{C}(\mathrm{OH})_{3}^{+}$cations, respectively. The acceptor group is in all cases the same cytosine-like fragment formed after the initial addition of RSH making $\mathrm{C} 5, \mathrm{sp}^{3}$ hybridized under protonation. The C5 atom is the crucial atom for the $\pi$-electron delocalization to the acceptor group in its $\mathrm{sp}^{2}$ hybridization state. After $\mathrm{sp}^{3}$ hybridization (protonation) this displacement is suppressed resulting in favoring carbonyl hydration of the formyl and carboxyl group in X. De facto this approach (also) explains the differences in the mechanistic views for the $\mathrm{C}-\mathrm{C}$ bond cleavage conversion rate cadC $>$ $\mathrm{fdC}>>$ hmdC. Under dissociation of the $\mathrm{HC}(\mathrm{OH})_{2}^{+}$and $\mathrm{C}(\mathrm{OH})_{3}^{+}$cations the $\mathrm{C} 5=\mathrm{C} 6$ double bond is restored under leaving of the thiol anion.

However, before protonation the formyl and carboxyl substituents may interact via their $\pi$-electron acceptance tendency with the donating N3-C4-C5 anionic fragment as shown in Figure 4. This results in lowering of the $\mathrm{C} 5$ proton affinity. In the study of Raiber et al., it was shown that the hydrogen bond between the exocyclic amino group on C4 and the carbonyl group of the formyl substituent results in a single conformation of the bond linking the $\mathrm{C} 5$ and $\mathrm{C}$ (formyl) groups [6]. More recently, a similar study was published by Wang et al. for 5-formylcytidine, as previously discovered in the mitochondrial tRNA of many species including human, indicating that in RNA 5-formylcytidine also plays an epigenetic role. They found that the 5-formyl group could increase duplex thermal stability and enhance base-pairing specificity. This was shown for an X-ray of a self-complementary octamer RNA duplex

5'-GUA(5fC)GUAC-3'. The results showed that the 5-formyl group is located in the same plane as the cytosine base with an intra hydrogen bond of the formyl group with the exocyclic amino group on the C4 position, suggesting an agreement with the corresponding 5fC-containing DNA [26].

From both studies it seems clear that the carbonyl group is in-plane with cytosine. On the other hand this conclusion is based on a crystallographic observation that may be at variance with the representation under dynamic conditions. In the out-of-plane of the carbonyl group the basicity of $\mathrm{C} 5$ increases. On the other hand if the oxygen of the carbonyl group is protonated in consequence of its planar arrangement then via a keto-enol equilibrium $\mathrm{C} 5$ is still the preferred location for the proton.

\subsection{Watson and Crick Hydrogen-Bond Bridging in Exploring New DNA-Like Duplex Structures}

Generally, research concerning Watson-Crick modelling is based on variation in sugar and base structures, and backbone conformations. As a new perspective we use the duplex structure as shown in Figure 2 and take the fragment as indicated in Figure 6 showing a new frame for a (possible) duplex structure as abstracted from the bridging in a $\mathrm{C}-\mathrm{G}$ pair. 


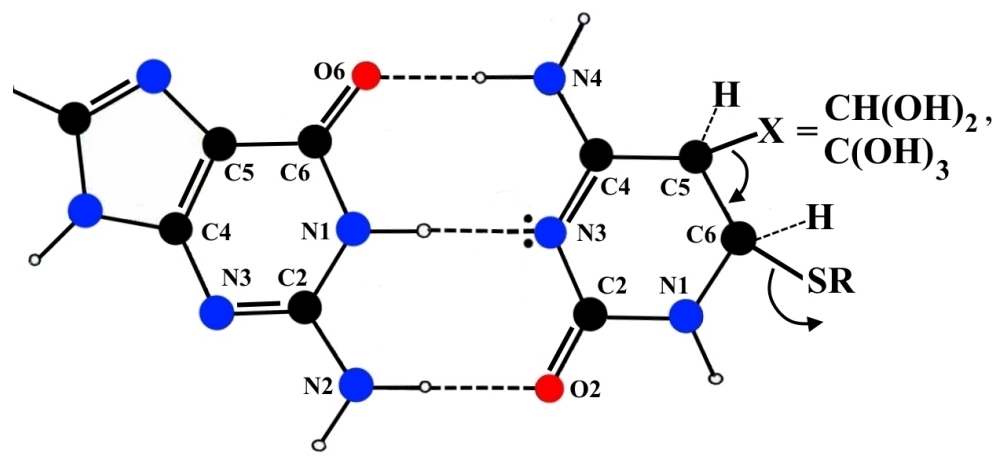

Figure 5. Reactive intermediate of the $\mathrm{C}-\mathrm{C}$ bond cleavage in $\mathrm{fdC}$ and cadC in which $\mathrm{X}$ is replaced by short-living $\mathrm{CH}(\mathrm{OH})_{2}$ and $\mathrm{C}(\mathrm{OH})_{3}$ groups after hydration of the formyl and carboxyl group, respectively. The formation of the original situation by restoring the $\mathrm{C} 5=\mathrm{C} 6$ bond has been demonstrated by electron displacement as indicated.

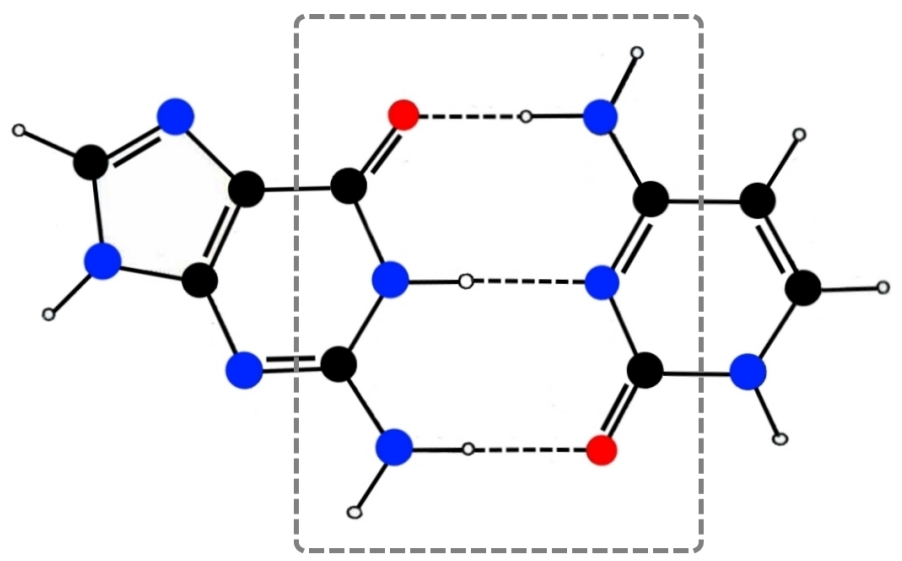

Figure 6. Representation of the frame for a new duplex structure as derived from the hydrogen bridging of the Watson and Crick C-G pair.

So there are three duplex structures involved based on the locations of hydrogen between the nitrogens as shown in Figure 7.

Based on the arrangement of the participating atoms the center structure has $\mathrm{C}_{2 \mathrm{~h}}$ symmetry. Taken for the N-H bond $1.06 \AA$, we derived for the distance of the symmetric center N---H---N bonding $1.413 \AA$ (the N-N distance of $2.827 \AA$ is in excellent agreement with corresponding distances in DNA).These calculations are based on the specific dynamics of the tetrahedral concept of Van't Hoff in the case of reactions with inversion of the reactive center [27]. The formation of an intermediate or transition complex with $\mathrm{C}_{2 h}$ symmetry is so far of theoretical interest.

In a combined experimental, theoretical and Van't Hoff dynamics for identity methyl, proton, hydrogen atom and hydride exchange reactions the correlation with three-center four-, three-, and two-electron systems was clearly demonstrated. It may be possible that under selected conditions hydrogen-bridging symmetrization in combination with the presence of highly symmetric elements may be experimentally verified. For that purpose Figure 7 is illustrative. In this 


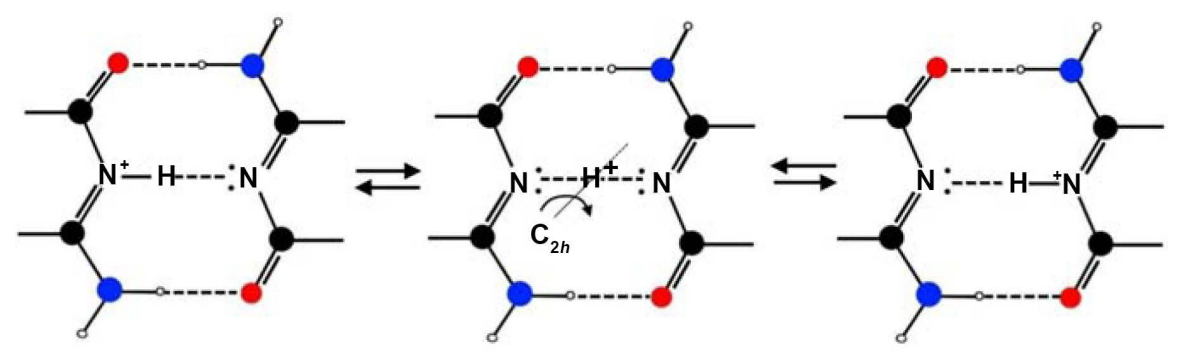

Figure 7. The three duplex structures as abstracted from Figure 6.

representation only the location of the imino proton in the central position is uncertain with respect to the other atomic positions, vide infra. The results deal with a stationary point on the reaction coordinate for proton transfer. The bond distances $(d)$ for a symmetrically transition state $(T S)$ can be obtained with:

$$
d(T S)=[1+n / 12] \times d(\text { initial state })
$$

in which $n$ is the number of electrons in the three-atom bonding: 4 (proton center), 3 (hydrogen atom center), and 2 (hydride center). In fact this reaction coordinate is the same coordinate for the nucleophilic displacement in the classical $\mathrm{S}_{\mathrm{N}} 2$ reactions, i.e. the introduced Van't Hoff dynamics. The comparison in distances with highly sophisticated $a b$ initio calculations differ (even) in a positive manner for axial ligand atoms possessing extended electron configurations. The latter aspect is one of the serious problems in the $a b$ initio approach.

In exploring duplex structures with this specific symmetry element, we took the C-G pair by eliminating the NCN fragment of the five-membered ring of guanine in order to restrict our model description to 6-membered rings. Under protonation of $\mathrm{G}$, lone-pair transfer of the central nitrogen occurs to the other nitrogen in the same ring under formation of a new N-H bond. The procedure is given in Figure 8. Formally, the result can be described as a protonation of CG at the $\mathrm{N} 3$ position of $\mathrm{G}$ remaining $\mathrm{N} 1$ positively charged.

From the different geometries with their inversion center for the three hydrogen bridges we can construct parallel and antiparallel DNA-like duplexes. If both rings and the hydrogen-bridging atoms are connected with the same inversion center then we are dealing with a parallel duplex. In the other case when both rings are connected with a plane of symmetry (mirror plane) in combination with the inversion center for the hydrogen-bridging atoms then an antiparallel duplex is formed. In the case of Figure 8 we are thus dealing with a dimer accommodating an antiparallel structure. It is of importance to mention that the lone-pair transfer from $\mathrm{N} 1$ to $\mathrm{N} 3$ (in the same ring) as indicated, leads to a formal positive charge on $\mathrm{N} 1$ that promotes the acidity of the hydrogen at that position.

This circumstance may strengthen exchange of the (central) imino proton between $\mathrm{G}$ and $\mathrm{C}$ as suggested before. The other example is given for the cytosine-cytosine base pair under acidic condition. Here we are dealing with an overall symmetry that both rings and the hydrogen-bridging atoms are connected 


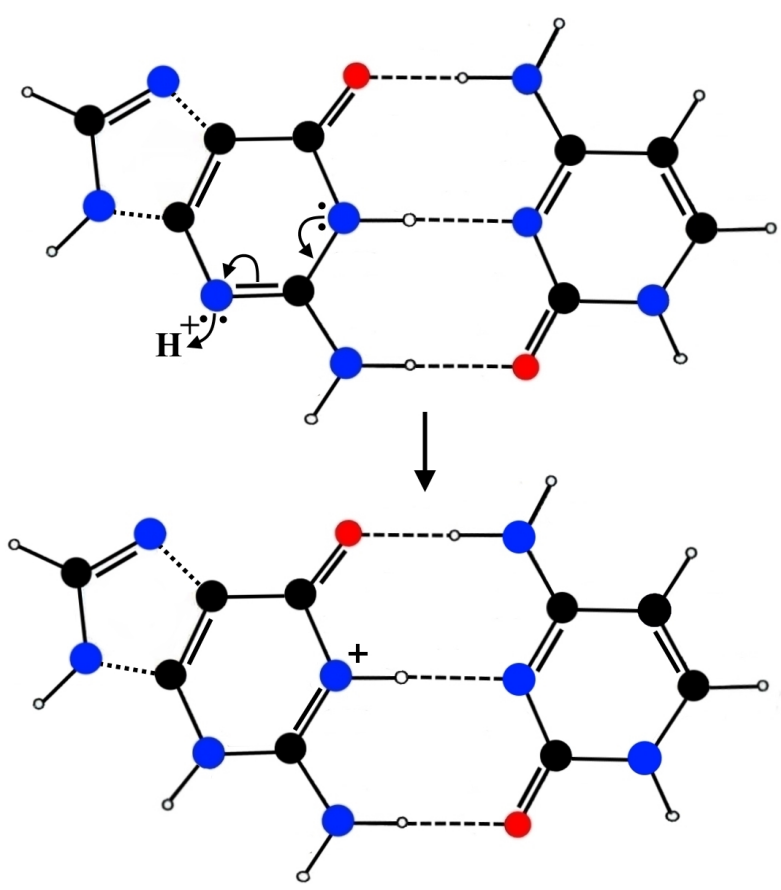

Figure 8. Lone-pair transfer of the central nitrogen in guanine to the other nitrogen in the same ring under formation of a new N-H bond. The 5-membered ring in guanine is indicated as insertion.

with the same inversion center that accommodates a parallel structure. The result is given in Figure 9.

Such a kind of interaction may be found in natural duplex DNA with C-rich sequences. Even in weakly acidic media a regiospecific dissociation of the $\mathrm{C}$-rich strand from the complementary $\mathrm{G}$ strand occurs accompanied with duplex formation of the $\mathrm{C}$ bases and the N3-protonated ones in a parallel arrangement as shown in Figure 9, demonstrating the key role of the inversion center realized by protonation of $\mathrm{N} 3$ as the result of the weak hydrogen bonding between $\mathrm{N} 1-\mathrm{H}$ and N3 shown for CG pairing in Figure 2. The example is unique in comparison with the other bases. This generalization is based partly on research as given below.

Sarma et al. demonstrated a cytosine-cytosine base pair at pH equals 3 [28]. As mentioned before, in the case of phosphate methylation we found a parallel DNA via two symmetry-related N4-H---N3 hydrogen bonds exclusively for the $S$ configuration of phosphorus [1] [29]. A similar observation has been done for natural DNA dC oligomers in the presence of selected cationic oligopeptides as supported by molecular mechanics calculations [15].

Later Gehring et al. described with NMR a tetrameric DNA structure with protonated cytosine-cytosine base pairs [30]. They solved the structure of the DNA oligomer 5'-d(TCCCCC)-3' at acid $\mathrm{pH}$ and concluded that the four-stranded complex with two base-paired parallel-stranded duplexes are closely associated with their bases intercalated. The structure is highly symmetrical on the NMR 


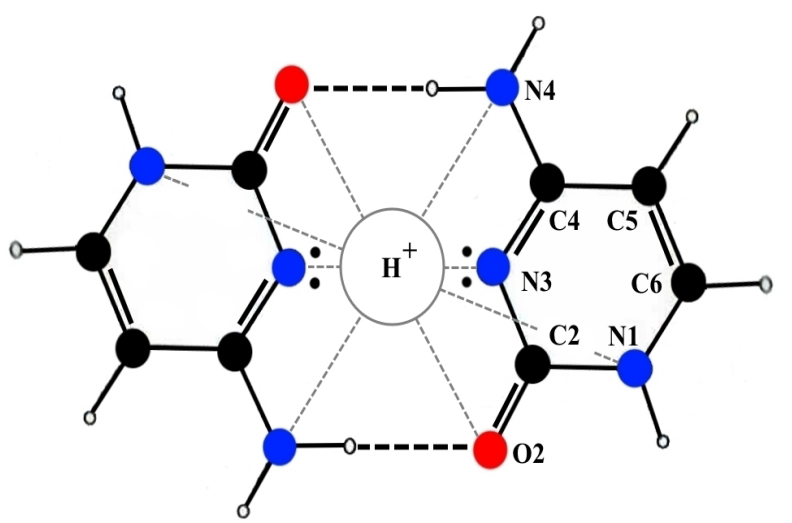

Figure 9. The proposed structure for the cytosine-cytosine base pair under acidic condition. The inversion center is indicated with the circled proton between the $\mathrm{N} 3$ atoms.

time scale. The best lower bond for the jumping rate of the imino proton is greater than $8 \times 10^{4} \mathrm{~s}^{-1}$. Lieblein et al. published a NMR study with quantum chemical calculations on these DNA $i$-motif structures of cytosine-cytosine base pair under acidic conditions [31]. The imino proton exchange was described as an asymmetric double-well potential with a proton hopping rate of at least $10^{8} \mathrm{~s}^{-1}$.

Buck et al. discussed the specific role of the proton in the central position between the cytosine-cytosine base pairs. It is clear that the extra proton will reduce the repulsion between the phosphate groups. Besides attention was given to the biological relevance of 2'-deoxy-3',5'-di- $O$-acetyl guanosine because of its conformational correspondence with the guanosine quadruplex. The latter hydrogen bonding is exclusively found in the parallel formation of thymine-thymine pairing [1].

Recently there is in-vivo evidence for such cytosine-rich regions in the genome as was reported by Zeraati et al. [32]. They demonstrated that the $i$-motif structures have a key regulatory role in the genome.

Many interesting suggestions can be made in this field. We suggest an application based on the inverted hydrogen-bridging structure in relation to inhibition of genetic information on DNA and RNA level.

\subsection{A New Model for Inhibition of Genetic Transfer}

In this model we took profit from the duplex structures as shown in Figure 6.

This specific hybridization for $\mathrm{G}$ and $\mathrm{C}$ has been developed on the basis of the hydrogen bridging between these bases. This selected site for hybridization can be recognized on DNA and RNA level. In the case of G the inhibitor (Y) is uncharged whereas for $\mathrm{C}$ we are dealing with the corresponding salt form $\left(\mathrm{YH}^{+}\right)$. In the latter form there may be an extra stabilization through the protonated central nitrogen as shown in Figure 10. The option for $\mathrm{X}$ depends on the implementation of chosen experiments. Comparison with other models for inhibition on selected sequences of natural DNA and RNA systems is not feasible because in these cases the main idea was based on modifications of the sugar ring [18] 


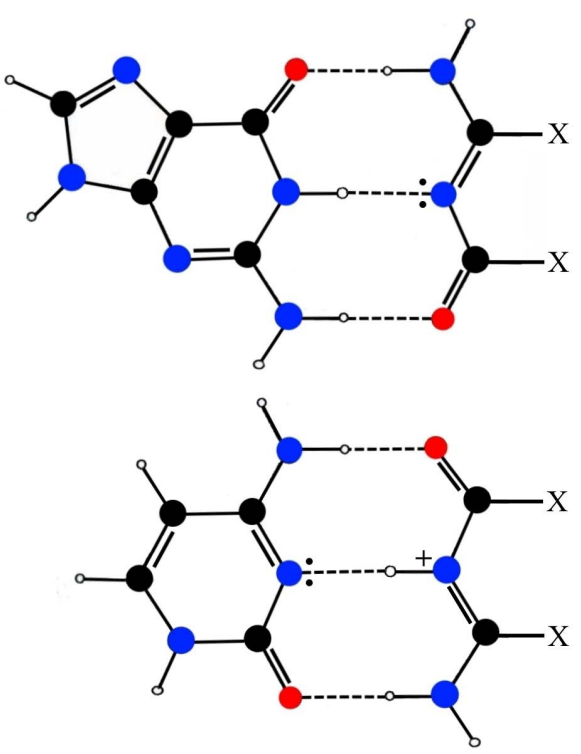

Figure 10. Hybridization of guanine (top) and cytosine (bottom) with a five-atom system indicated in the text as $\mathrm{Y}$ (top) and $\mathrm{YH}^{+}$(bottom), respectively. $\mathrm{X}$ stands for a selected choice.

and the backbone as phosphate methylated [1] [7] [10] [14]. It is tempting to suggest many possibilities for further studies as solving the molecular structure for $[\mathrm{G} . \mathrm{Y}]_{n},\left[\mathrm{C} . \mathrm{YH}^{+}\right]_{n}$, and mixed combinations on the DNA and RNA level with the usual hybridization technology. It is to be expected that a single strand of $\mathrm{dG}_{n}$ and $\mathrm{dC}_{n}$ or mixed combinations will exhibit some degree of secondary structure either by transient stacking or by base pairing in self complementary regions (mixed structure) especially focused on the impact of the inhibitors on the overall structure. We suppose that the structures of $\mathrm{Y}$ and $\mathrm{YH}^{+}$will accommodate the planar structures of $\mathrm{G}$ and $\mathrm{C}$, respectively.

Summarizing, in one part of the double stranded helix the stacking is absent which might be suggest flexibility favoring conformational transitions reflecting intermediates of the DNA in vivo dynamics.

During our research we came aware of a somewhat similar duplex formation in comparison with the structure given in Figure 7. This self-assembled dimer complex was based on a quadruple hydrogen bonding [33]. The X-ray structure is given in Figure 11.

\section{Conclusion}

The epigenetic elements 5-hydroxymethyl-dC, 5-formyl-dC, and 5-carboxy-dC may be considered as intermediates of an active demethylation process. A detailed mechanistic and theoretical model based on sophisticated ab initio calculations is given for the $\mathrm{C}-\mathrm{C}$ bond cleavage as one of the possibilities for the reconversion of $\mathrm{dC}$. This approach focused on the chemistry within the DNA duplex structure. There is a clear spin-off chemistry of this approach in evaluating new duplex systems related to the Watson-Crick duplexes concerning their 


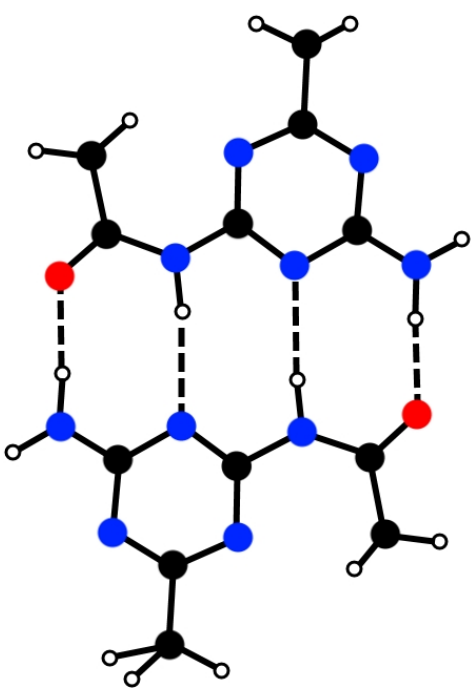

Figure 11. Self-assembled dimer complex through quadruple hydrogen bonding.

hydrogen-bond symmetrization [34]. A model is composed for a base-specific inhibition of G and C on the DNA and RNA level. This approach may be considered as an exclusive model within the synthetic biology.

\section{Acknowledgements}

I thank Professor Anthony Spek of the Bijvoet Center of Biomolecular Research (Utrecht) for the X-ray data of the self-assembled dimer. I thank my grandson Robin van Dorrestein for his valuable technical assistance.

\section{Conflicts of Interest}

The author declares no conflicts of interest regarding the publication of this paper.

\section{References}

[1] Buck, H.M. (2011) DNA Systems for B-Z Transitions and Their Significance as Epigenetic Model: The Fundamental Role of the Methyl Group. Nucleosides, Nucleotides and Nucleic Acids, 30, 918-944. https://doi.org/10.1080/15257770.2011.620580

[2] Kriaucionis, S. and Heintz, N. (2009) The Nuclear DNA Base 5-Hydroxymethylcytosine Is Present in Purkinje Neurons and the Brain. Science, 324, 929-93. https://doi.org/10.1126/science.1169786

[3] Globisch, D., Münzel, M., Müller, M., Michalakis, S., Wager, M., Koch, S., Brückl, T., Biel, M. and Carell, T. ( 2010) Tissue Distribution of 5-Hydroxymethylcytosine and Search for Active Demethylation Intermediates. PLoS ONE, 5, e15367. https://doi.org/10.1371/journal.pone.0015367

[4] Münzel, M., Globisch, D. and Carell, T. (2011) 5-Hydroxymethyl-Cytosine, the Sixth Base of the Genome. Angewandte Chemie International Edition, 50, 6460-6468. https://doi.org/10.1002/anie.201101547

[5] Wang, S.R., Long, Y.L., Wang, J.Q., Ge, Y.S., Guo, P., Liu, Y., Tian, T. and Zhou, X. (2014) Systematic Investigations of Different Cytosine Modifications on CpG Di- 
nucleotide Sequences: The Effects on the B-Z Transition. Journal of the American Chemical Society, 136, 56-59. https://doi.org/10.1021/ja4107012

[6] Raiber, E.-A., Murat, P., Chirgadze, D.Y., Beraldi, D., Luisi, B.F. and Balasubramanian, S. (2015) 5-Formylcytosine Alters the Structure of the DNA Double Helix. Nature Structural \& Molecular Biology, 22, 44-49. https://doi.org/10.1038/nsmb.2936

[7] Buck, H.M. (2004) The Chemical and Biochemical Properties Methylphosphotriester DNA. Nucleos. Nucleotides and Nucleic Acids, 23, 1833-1847. https://doi.org/10.1081/NCN-200040620

[8] Pfaffeneder, T., Hackner, B., Truss, M., Müller, M., Deiml, C.A., Hagemeier, C. and Carell, T. (2011) The Discovery of 5-Formylcytosine in Embryonic Stem Cell DNA. Angewandte Chemie International Edition, 50, 7008-7012. https://doi.org/10.1002/anie.201103899

[9] Iwan, K., Rahimoff, R., Kirchner, A., Spada, F., Schröder, A.S. ,Kosmatchev, O., Ferizai, S., Steinbacher, J., Parsa, E., Müller, M. and Carell, T. (2018) 5-Formylcytosine to Cytosine Conversion by C-C Bond Cleavage in Vivo. Nature Chemical Biology, 14, 72-78. https://doi.org/10.1038/nchembio.2531

[10] Buck, H.M. (2013) A Conformational B-Z DNA Study Monitored with Phosphatemethylated DNA as a Model for Epigenetic Dynamics Focused on 5-(Hydroxy) Methylcytosine. Journal of Biophysical Chemistry, 4, 37-46. https://doi.org/10.4236/jbpc.2013.42005

[11] Fonseca Guerra, C. (2000) Structure and Bonding of DNA. Ph.D. Thesis, Free University of Amsterdam, Amsterdam.

[12] Schiesser, S., Pfaffeneder, T., Sadeghian, K., Hackner, B., Steigenberger, B., Schröder, A.S., Steinbacher, J., Kashiwazaki, G., Höfner, G., Wanner, K.T., Ochsenfeld, C. and Carell, T. (2013) Deamination, Oxidation, and C-C Bond Cleavage Reactivity of 5-Hydroxymethylcytosine, 5-Formylcytosine, and 5-Carboxycytosine. Journal of the American Chemical Society, 135, 14593-14599. https://doi.org/10.1021/ja403229y

[13] Fonseca Guerra, C., Bickelhaupt, F.M., Snijders, J.G. and Baerends, E.-J. (2000) Hydrogen Bonding in DNA Base Pairs. Reconciliation of Theory and experiment. Journal of the American Chemical Society, 122, 4117-4128. https://doi.org/10.1021/ja993262d

[14] Buck, H.M. (2016) Modified RNA with a Phosphate-Methylated Backbone. A Serious Omission in Our (Retracted) Study at HIV-1 RNA Loops and Integrated DNA. Specific Properties of the (Modified) RNA and DNA Dimers. Journal of Biophysical Chemistry, 7, 30-44. https://doi.org/10.4236/jbpc.2016.71003

[15] Van Genderen, M.H., Hilbers, M.P., Koole, L.H. and Buck, H.M. (1990) Peptide-Induced Parallel DNA Duplexes for Oligopyrimidines. Stereospecificity in Complexation for Oligo(L-Lysine) and Oligo(L-Ornitine). Biochemistry, 29, 7838-7845. https://doi.org/10.1021/bi00486a009

[16] Corey, D.R. (2017) Nusinersen, an Antisence Oligonucleotide Drug for Spinal Muscular Atrophy. Nature Neuroscience, 20, 497-499. https://doi.org/10.1038/nn.4508

[17] Knouse, K.W., deGruyter, J.N., Schmidt, M.A., Zheng, B., Vantourout, J.C., Kingston, C., Mercer, S.E., Mcdonald, I.M., Olson, R.E., Zhu, Ye., et al. (2018) Unlocking $\mathrm{P}(\mathrm{V})$ : Reagents for Chiral Phosphorothioate Synthesis. Science, 361, 1234-1238. https://doi.org/10.1126/science.aau3369

[18] Lin, Q.-Y., Mason, J.A., Li, Z., Zhou, W., O’Brien, M.N., Brown, K.A., Jones, M.R., 
Butun, S., Lee, B., Dravid, V.P., Avdin, K. and Mirkin, C.A. (2018) Building Superlattices from Individual Nanoparticles via Template-Confined DNA-Mediated Assembly. Science, 359, 669-672. https://doi.org/10.1126/science.aaq0591

[19] Tardy-Planechaud, S., Fujimoto, J., Lin, S.S. and Sowers, L.C. (1997) Solid Phase Synthesis and Restriction Endonuclease Cleavage of Oligodeoxynucleotides Containing 5-(Hydroxymethyl)-Cytosine. Nucleic Acids Research, 25, 553-559. https://doi.org/10.1093/nar/25.3.553

[20] Dai, Q. and He, C. (2011) Synthesis of 5-Formyl- and 5-Carboxyl-dC Containing DNA Oligos as Potential Oxidation Products of 5-Hydroxymethylcytosine in DNA. Organic Letters, 13, 3446-3449. https://doi.org/10.1021/ol201189n

[21] Münzel, M., Lischke, U., Stathis, D., Pfaffeneder, T., Gnerlich, F.A., Deiml, C.A., Koch, S.C., Karaghiosoff, K. and Carell, T. (2011) Improved Synthesis and Mutagenicity of Oligonucleotides Containing 5-Hydroxymethylcytosine, 5-Formylcytosine and 5-Carboxylcytosine. Chemistry: A European Journal, 17, 13782-13788. https://doi.org/10.1002/chem.201102782

[22] Steigenberger, B., Schiesser, S., Hackner, B., Brandmayr, C., Laube, S.K., Steinbacher, J., Pfaffeneder, T. and Carell, T. (2013) Synthesis of 5-Hydroxymethyl-, 5-Formyl-, and 5-Carboxycytidine-Triphosphates and Their Incorporation into Oligonucleotides by Polymerase Chain Reaction. Organic Letters, 15, 366-369. https://doi.org/10.1021/ol3033219

[23] Schiesser, S., Hackner, B., Pfaffeneder, T., Müller, M., Hagemeier, C., Truss, M. and Carell, T. (2012) Mechanism and Stem-Cell Activity of 5-Carboxycytosine Decarboxylation Determined by Isotope Tracing. Angewandte Chemie International Edition, 51, 6516-6520. https://doi.org/10.1002/anie.201202583

[24] Errea, I., Calandra, M., Pickard, C.J., Nelson, J.R., Needs, R.J., Li, Y., Liu, H., Zhang, Y., Ma, Y. and Mauri, F. (2016) Quantum Hydrogen-Bond Symmetrization in the Superconducting Hydrogen Sulfide System. Nature, 532, 81-84. https://doi.org/10.1038/nature17175

[25] Buck, H.M. (2017) A Molecular Description of Superconductivity of Sulfur Hydride and Related Systems under High-Pressure Conditions. Open Journal of Physical Chemistry, 7, 9-25. https://doi.org/10.4236/ojpc.2017.71002

[26] Wang, R., Luo, Z., He, K., Delaney, M.O., Chen, D. and Sheng, J. (2016) Base Pairing and Structural Insights into 5-Formylcytosine in RNA Duplex. Nucleic Acids Research, 44, 4968-4977. https://doi.org/10.1093/nar/gkw235

[27] Buck, H.M. (2008) A Combined Experimental, Theoretical, and Van't Hoff Model Study for Identity, Methyl, Proton, Hydrogen Atom, and Hydride Exchange Reactions. International Journal of Quantum Chemistry, 108, 1601-1614. https://doi.org/10.1002/qua.21683

[28] Sarma, M.H., Gupta, G. and Sarma, R.H. (1986) A Cytosine-Cytosine Base Paired Parallel DNA Double Helix with Thymine-Thymine Bulges. FEBS Letters, 205, 223-229. https://doi.org/10.1016/0014-5793(86)80902-4

[29] Quaedflieg, P.J.L.M., Broeders, N.L.H.L., Koole, L.H., van Genderen, M.H.P. and Buck, H.M. (1990) Conformation of the Phosphate-Methylated DNA Dinucleotides $\mathrm{d}\left(\mathrm{C}_{\mathrm{P}} \mathrm{G}\right)$ and $\mathrm{d}\left(\mathrm{T}_{\mathrm{P}} \mathrm{C}\right)$. Formation of a Parallel Miniduplex Exclusively for the $S$ Configuration at Phosphorus. The Journal of Organic Chemistry, 55, 122-127. https://doi.org/10.1021/jo00288a025

[30] Gehring, K., Leroy, J.-L. and Guéron, M. (1993) A Tetrameric DNA Structure with Protonated Cytosine-Cytosine Base Pairs. Nature, 363, 561-565. https://doi.org/10.1038/363561a0 
[31] Lieblein, A.L., Krämer, M., Dreuw, A., Fürtig, B. and Schwalbe, H. (2012) The Nature of Hydrogen Bonds in Cytidine $\mathrm{H}^{+}$Cytidine DNA Base Pairs. Angewandte Chemie International Edition in English, 51, 4067-4077.

https://doi.org/10.1002/anie.201200549

[32] Zeraati, M., Langley, D.B., Schofield, P., Moye, A.L., Rouet, R., Hughes, W.E., Bryan, T.M., Dinger, M.E. and Christ, D. (2018) I-motif DNA Structures Are Formed in the Nuclei of Human Cells. Nature Chemistry, 10, 631-637.

https://doi.org/10.1038/s41557-018-0046-3

[33] Beijer, F.H., Kooijman, H., Spek, A.L., Sijbesma, R.P. and Meijer, E.W. (1998) Self-Complementarity Achieved through Quadruple Hydrogen Bonding. Angewandte Chemie International Edition in English, 37, 75-78.

https://doi.org/10.1002/(SICI)1521-3773(19980202)37:1/2<75::AID-ANIE75>3.0.C $\mathrm{O} ; 2-\mathrm{R}$

[34] Mo, Y. (2006) Probing the Nature of Hydrogen Bonds in DNA Base Pairs. Journal of Molecular Modeling, 13, 665-672. https://doi.org/10.1007/s00894-005-0021-y 